\title{
Regressor and Disturbance Have Moments of All Order, Least Squares Estimator Has None
}

\author{
Kenneth D. West ${ }^{1}$ \\ University of Wisconsin \\ Zifeng Zhao ${ }^{2}$ \\ University of Wisconsin
}

March 2016

\begin{abstract}
We construct an example in which the least squares estimator has unbounded bias and no moments, even though the regressor and disturbance have moments of all order and are independently distributed across observations.
\end{abstract}

Keywords: least squares bias; mean squared error; negative moments.

We thank two referees for helpful comments. West thanks the National Science Foundation for financial support.

1 Department of Economics, University of Wisconsin. Email: kdwest@wisc.edu

${ }^{2}$ Corresponding author. 1300 University Avenue, Department of Statistics, University of Wisconsin-Madison, Madison, WI 53706. Email: zifeng.zhao@wisc.edu 


\section{Introduction}

In this note, we construct a univariate example in which the least squares estimator has unbounded bias and no moments, even though the regressor and disturbance have moments of all order and are independently distributed across observations. As well, the regressor is continuously distributed, so our example does not turn on a possible inability to invert the moment matrix of the regressor.

Our example has two motivations. The first comes from consideration of bias or mean squared prediction error in least squares regressions. Let $X$ be the $T \times k$ design matrix, where $T$ is the sample size and $k$ the number of regressors. Then $X^{\prime} X$ is the $k \times k$ moment matrix of the regressors. To insure integrability of the least squares estimator, literature on least squares bias or mean squared prediction error sometimes makes a high level assumption on the existence of moments of $\left(X^{\prime} X\right)^{-1}$ (e.g., Shaman (2010)) or implicitly assumes integrability without making a specific assumption (e.g., Bao and Ullah (2010)). Our paper contributes by illustrating that innocuous assumptions about dependence and moments do not suffice to insure integrability. The second motivation relates

to the existence of negative moments. Research has given a necessary and sufficient condition for the existence of a negative first moment for a given random variable (Khuri and Casella (2002)) and, in the least squares context, a sufficient condition for existence of moments of $\left(X^{\prime} X\right)^{-1}$ (Findley and Wei (2002)). Our paper contributes by supplying a weaker sufficent condition than in Findley and Wei (2002), although ours, unlike Findley and Wei's, is only applicable for a univariate regression setup.

In section 2, we give a necessary and sufficient condition for the existence of moments of $\left(X^{\prime} X\right)^{-1}$ for a univariate regression model. We then present a sufficient condition for the existence of moments of the least square estimator. In section 3, we construct a counterexample in which the least squares estimator has unbounded bias and no moments, even though the regressor and disturbance have moments of all order. Section 4 has some comments.

\section{Lemmas on the existence of moments}

Write the regression as

$$
y_{t}=x_{t} \beta+\eta_{t}, t=1, \ldots T .
$$

where all variables are scalars; $y_{t}$ and $x_{t}$ are observed, $\eta_{t}$ is the unobservable disturbance, and $\beta$ is the parameter of interest. Thus the least squares estimator is $\hat{\beta}_{T}=\left(\sum_{t=1}^{T} x_{t}^{2}\right)^{-1}\left(\sum_{t=1}^{T} x_{t} y_{t}\right)=\beta+\left(\sum_{t=1}^{T} x_{t}^{2}\right)^{-1}\left(\sum_{t=1}^{T} x_{t} \eta_{t}\right)$. (We omit the usual $\frac{1}{T}$ normalization of each of the sums to keep notation uncluttered.) In the notation of the introduction, $k=1$ and $\sum_{t=1}^{T} x_{t}^{2}$ corresponds to $X^{\prime} X$. 
From Holder's inequality, for any $\varepsilon>0$,

$$
\left|E\left(\hat{\beta}_{T}-\beta\right)\right| \leq\left[E\left|\left(\sum_{t=1}^{T} x_{t}^{2}\right)^{-1}\right|^{(1+\varepsilon)}\right]^{\frac{1}{1+\varepsilon}} \times\left[E\left|\left(\sum_{t=1}^{T} x_{t} \eta_{t}\right)\right|^{\frac{1+\varepsilon}{\varepsilon}}\right]^{\frac{\varepsilon}{1+\varepsilon}} .
$$

Hence if $x_{t}$ and $\eta_{t}$ have moments of all order (as we shall shortly assume), a necessary condition for $E\left|\hat{\beta}_{T}-\beta\right|=\infty$ is that $\left(\sum_{t=1}^{T} x_{t}^{2}\right)^{-1}$ has no moment of order higher than one, and a sufficient condition for $E\left|\hat{\beta}_{T}-\beta\right|<\infty$ is that there exists $\varepsilon>0$ s.t. $E\left(\sum_{t=1}^{T} x_{t}^{2}\right)^{-(1+\varepsilon)}<\infty$.

Intuitively, in order for $E\left(\sum_{t=1}^{T} x_{t}^{2}\right)^{-1}=\infty$, one wants $\left(\sum_{t=1}^{T} x_{t}^{2}\right)^{-1}$ to have a fat tail, i.e., one wants $\sum_{t=1}^{T} x_{t}^{2}$ to have high probability of being near zero. The following Lemma 1 is central to our construction of $x_{t}$ that satisfies this criterion, delivering a sufficient and necessary condition for $\left(\sum_{t=1}^{T} x_{t}^{2}\right)^{-1}$ to have moments of order $s$.

Lemma 1: Let $\delta_{t}$ be a sequence of parameters, $0<\delta_{t}<1, t=1,2, \ldots T$. For a sequence of independent random variables $z_{t}, t=1, \ldots, T$, let the cumulative distribution function (cdf) of $z_{t}$ be $P\left(z_{t} \leq z\right)=z^{\delta_{t}}, 0<z \leq 1$. Then for any $s>0$, a sufficient and necessary condition for $E\left(\sum_{t=1}^{T} z_{t}\right)^{-s}=\infty$ is $\sum_{t=1}^{T} \delta_{t} \leq s$ for some $s>0$.

Proof: To show $\sum_{t=1}^{T} \delta_{t} \leq s \Rightarrow E\left(\sum_{t=1}^{T} z_{t}\right)^{-s}=\infty$, let $c>0$ be an arbitrary positive constant. By Markov's inequality,

$E\left(\frac{1}{\sum_{t=1}^{T} z_{t}}\right)^{s} \geq E\left(\frac{1}{\left(\sum_{t=1}^{T} z_{t}\right)^{s}} \cdot I\left(\frac{1}{\left(\sum_{t=1}^{T} z_{t}\right)^{s}}>c\right)\right) \geq P\left[\frac{1}{\left(\sum_{t=1}^{T} z_{t}\right)^{s}} \geq c\right] \cdot c$. 
Further,

$$
\begin{aligned}
& P\left[\frac{1}{\left(\sum_{t=1}^{T} z_{t}\right)^{s}} \geq c\right] \cdot c=P\left[\sum_{t=1}^{T} z_{t} \leq \frac{1}{c^{1 / s}}\right] \cdot c \\
& \geq c \times P\left(z_{1} \leq \frac{1}{2 c^{1 / s}}, z_{2} \leq \frac{1}{2^{2} c^{1 / s}}, \cdots, z_{T} \leq \frac{1}{2^{T} c^{1 / s}}\right) \\
& =c \times P\left(z_{1} \leq \frac{1}{2 c^{1 / s}}\right) P\left(z_{2} \leq \frac{1}{4 c^{1 / s}}\right) \cdots P\left(z_{T} \leq \frac{1}{2^{T} c^{1 / s}}\right) \\
& =c\left(\frac{1}{2 c^{1 / s}}\right)^{\delta_{1}}\left(\frac{1}{2^{2} c^{1 / s}}\right)^{\delta_{2}} \cdots\left(\frac{1}{2^{T} c^{1 / s}}\right)^{\delta_{T}}=2^{-\sum_{t=1}^{T} t \delta_{t}} \cdot c^{1-\sum_{t=1}^{T} \delta_{t} / s} .
\end{aligned}
$$

The equality on the third line follows from independence of the $z_{t}$ 's.

For $\sum_{t=1}^{T} \delta_{t}<s$, since $c$ can be made arbitrarily large, the desired result follows. For $\sum_{t=1}^{T} \delta_{t}=s$, by the above equation, we have

$$
E\left(\frac{1}{\left(\sum_{t=1}^{T} z_{t}\right)^{s}} \cdot I\left(\frac{1}{\left(\sum_{t=1}^{T} z_{t}\right)^{s}}>c\right)\right) \geq 2^{-\sum_{t=1}^{T} t \delta_{t}}, \quad \forall c>0,
$$

which is obviously not true if $E\left(\sum_{t=1}^{T} z_{t}\right)^{-s}<\infty$, so we have $E\left(\sum_{t=1}^{T} z_{t}\right)^{-s}=$ $\infty$.

The converse $\left(\sum_{t=1}^{T} \delta_{t}>s \Rightarrow E\left(\sum_{t=1}^{T} z_{t}\right)^{-s}<\infty\right)$ follows from an argument similar to one made in Sriram and Bose (1988) for constant $\delta$. Details are in the Appendix.

The key to Lemma 1 is that the cdf of $z_{t}$ is $z^{\delta_{t}}$ in a neighborhood of the origin (equivalently, the pdf (probability density function) of $z_{t}$ is $z^{\delta_{t}-1}$ in that neighborhood); the result follows even if we relax the condition to only require $P\left(z_{t} \leq z\right)=O\left(z^{\delta_{t}}\right)$ as $z \rightarrow 0$.

Using Lemma 1, we give a sufficient condition for $E\left|\hat{\beta}_{T}-\beta\right|<\infty$ :

Lemma 2: Let $\left\{\left(x_{t}, y_{t}\right)\right\}_{t=1}^{\infty}$ come from model (1) with observations are independent over time $t$. Assume that $x_{t}$ and $\eta_{t}$ have moments of all order and $\forall t, P\left(x_{t}^{2} \leq x\right)=O\left(x^{\delta_{t}}\right)$ as $x \rightarrow 0$, then we have $E\left|\hat{\beta}_{T}-\beta\right|<\infty$ for all $T$ such that $\sum_{t=1}^{T} \delta_{t}>1$. 
Proof: As mentioned above, since $\sum_{t=1}^{T} x_{t} \eta_{t}$ has moments of all order, by Holder's inequality, the sufficient condition for $E\left|\hat{\beta}_{T}-\beta\right|<\infty$ is that there exists an $\varepsilon>0$ s.t. $E\left(\sum_{t=1}^{T} x_{t}^{2}\right)^{-(1+\varepsilon)}<\infty$.

Since $\sum_{t=1}^{T} \delta_{t}>1$, we have that there exists an $\varepsilon>0$ s.t. $\sum_{t=1}^{T} \delta_{t}>1+\varepsilon$. By assumption, $\forall t, P\left(x_{t}^{2} \leq x\right)=O\left(x^{\delta_{t}}\right)$ as $x \rightarrow 0$, so by applying Lemma 1 at $s=1+\varepsilon$, we have $E\left(\sum_{t=1}^{T} x_{t}^{2}\right)^{-(1+\varepsilon)}<\infty$.

\section{A counterexample}

In this section, we shall construct an example in which least squares has unbounded bias, i.e., $E\left(\hat{\beta}_{T}-\beta\right)=\infty$, which implies as well that $\hat{\beta}_{T}$ has no moments.

Our process for $x_{t}$ in (1) will behave like $z_{t}$ when $x_{t}>0$, but when $x_{t}<0$ will have no mass in a neighborhood of zero. To simplify notation, our example sets $\theta_{t}=1-\delta_{t}$.

The joint distribution of $\left(x_{t}, \eta_{t}\right)$ in our example is as follows. Suppose $\left\{x_{t}, \eta_{t}\right\}_{t=1}^{\infty}$ are independent and not identically distributed (i.n.i.d.) random variables, where the pdf of $x_{t}$ 's is defined as:

$$
f_{t}(x)=\left\{\begin{array}{cl}
x^{-\theta_{t}} & \text { if } 0<x \leq c\left(\theta_{t}\right) \\
\frac{1}{2 K\left(\theta_{t}\right)} & \text { if }-\frac{5}{2} K\left(\theta_{t}\right)<x \leq-\frac{3}{2} K\left(\theta_{t}\right) \\
0 & \text { otherwise }
\end{array}\right.
$$

where $0<\theta_{t}<1$, and

$$
\int_{0}^{c\left(\theta_{t}\right)} x^{-\theta_{t}}=\frac{1}{2}, \text { i.e. } c\left(\theta_{t}\right)=\left(\frac{1-\theta_{t}}{2}\right)^{\frac{1}{1-\theta_{t}}},
$$

and

$$
K\left(\theta_{t}\right)=\int_{0}^{c\left(\theta_{t}\right)} x \cdot f_{t}(x) d x=E\left(x_{t} \cdot I\left(x_{t}>0\right)\right) .
$$

Thus, by construction $E\left(x_{t} \cdot I\left(x_{t}<0\right)\right)=-K\left(\theta_{t}\right)$. So we have $E\left(x_{t}\right)=0$ and median of $x_{t}$ is 0 . 
Based on $x_{t}$, the conditional distribution of $\eta_{t}$ is defined as

$$
\left\{\begin{array}{l}
\text { if } x_{t}>0, \quad P\left(\eta_{t}=\eta \mid x_{t}\right)=\left\{\begin{array}{cl}
p_{t} & \text { if } \eta=1 \\
1-p_{t} & \text { if } \eta=-x_{t}
\end{array}\right. \\
\text { if } x_{t}<0, \quad P\left(\eta_{t}=\eta \mid x_{t}\right)= \begin{cases}\frac{1}{2} & \text { if } \eta=c_{t} \\
\frac{1}{2} & \text { if } \eta=d_{t}\end{cases}
\end{array}\right.
$$

where non-stochastic $p_{t}, c_{t}$ and $d_{t}$ will be defined so that $E\left(\eta_{t}\right)=0$ and $E\left(\eta_{t} x_{t}\right)=0$. That is,

$$
\begin{aligned}
E\left(\eta_{t}\right) & =E\left(E\left(\eta_{t} \mid x_{t}\right)\right) \\
& =E\left(\left(p_{t}-\left(1-p_{t}\right) x_{t}\right) \cdot I\left(x_{t}>0\right)+\frac{c_{t}+d_{t}}{2} \cdot I\left(x_{t}<0\right)\right) \\
& =p_{t}-2\left(1-p_{t}\right) K\left(\theta_{t}\right)+\frac{c_{t}+d_{t}}{2}=0,
\end{aligned}
$$

and

$$
\begin{aligned}
E\left(\eta_{t} x_{t}\right) & =E\left(x_{t} E\left(\eta_{t} \mid x_{t}\right)\right) \\
& =E\left(\left(p_{t}-\left(1-p_{t}\right) x_{t}\right) \cdot x_{t} \cdot I\left(x_{t}>0\right)+\frac{c_{t}+d_{t}}{2} \cdot x_{t} \cdot I\left(x_{t}<0\right)\right) \\
& =p_{t} K\left(\theta_{t}\right)-\left(1-p_{t}\right) K_{2}\left(\theta_{t}\right)-\frac{c_{t}+d_{t}}{2} K\left(\theta_{t}\right)=0,
\end{aligned}
$$

where $K_{2}\left(\theta_{t}\right)=E\left(x_{t}^{2} \cdot I\left(x_{t}>0\right)\right)$.

Solving the above equations, we can easily get

$$
p_{t}=\frac{2 K\left(\theta_{t}\right)^{2}+K_{2}\left(\theta_{t}\right)}{2 K\left(\theta_{t}\right)^{2}+K_{2}\left(\theta_{t}\right)+2 K\left(\theta_{t}\right)}
$$

and

$$
c_{t}+d_{t}=4\left(1-p_{t}\right) K\left(\theta_{t}\right)-2 p_{t},
$$

and we choose arbitrary $c_{t}$ and $d_{t}$ such that $c_{t}+d_{t}$ satisfies this last equation.

In other words, for any sequence $\left\{\theta_{t}\right\}_{t=1}^{\infty}$ where $0<\theta_{t}<1 \forall t$, there exists $\left\{p_{t}, c_{t}, d_{t}\right\}_{t=1}^{\infty}$ such that $\left\{x_{t}, \eta_{t}\right\}_{t=1}^{\infty}$ has the distributions described above with $E\left(x_{t}\right)=0, E\left(\eta_{t}\right)=0$ and $E\left(\eta_{t} x_{t}\right)=0$ and median of $x_{t}$ is 0 .

Suppose we have (1), with $\hat{\beta}_{T}-\beta=\left(\sum_{t=1}^{T} x_{t}^{2}\right)^{-1}\left(\sum_{t=1}^{T} x_{t} \eta_{t}\right)$. In the following, for simplicity we set $\beta=0$. 
Theorem 1: Assume that $\sum_{t=1}^{T} \delta_{t}=\sum_{t=1}^{T}\left(1-\theta_{t}\right) \leq 1$, then we have

$$
E\left[\left(\hat{\beta}_{T}-\beta\right)^{+}\right]=E\left(\hat{\beta}_{T}^{+}\right)=\infty \text { and } E\left[\left(\hat{\beta}_{T}-\beta\right)^{-}\right]=E\left(\hat{\beta}_{T}^{-}\right)<\infty,
$$

so,

$$
E\left(\hat{\beta}_{T}-\beta\right)=E\left(\hat{\beta}_{T}\right)=E\left(\hat{\beta}_{T}^{+}\right)-E\left(\hat{\beta}_{T}^{-}\right)=\infty,
$$

where $\left(\hat{\beta}_{T}-\beta\right)^{+}=\max \left\{\hat{\beta}_{T}-\beta, 0\right\}$ and $\left(\hat{\beta}_{T}-\beta\right)^{-}=\max \left\{-\left(\hat{\beta}_{T}-\beta\right), 0\right\}$.

Proof: For the positive part,

$$
\begin{aligned}
& E\left(\hat{\beta}_{T}^{+}\right)=E\left(\frac{\sum_{t=1}^{T} \eta_{t} x_{t}}{\sum_{t=1}^{T} x_{t}^{2}}\right)^{+} \geq E\left(\frac{\left(\sum_{t=1}^{T} \eta_{t} x_{t}\right)^{+}}{\sum_{t=1}^{T} x_{t}^{2}} \cdot \prod_{t=1}^{T} I\left(x_{t}>0\right)\right) \\
& \geq E\left(\frac{\left(\sum_{t=1}^{T} \eta_{t} x_{t}\right)^{+}}{\sum_{t=1}^{T} x_{t}^{2}} \cdot \prod_{t=1}^{T} I\left(x_{t}>0\right) \cdot \prod_{t=1}^{T} I\left(\eta_{t}=1\right)\right)=E\left(\frac{\left(\sum_{t=1}^{T} \eta_{t} x_{t}\right)^{+}}{\sum_{t=1}^{T} x_{t}^{2}} \cdot \prod_{t=1}^{T} I\left(x_{t}>0, \eta_{t}=1\right)\right) \\
& =\int_{0}^{c\left(\theta_{T}\right)} \cdots \int_{0}^{c\left(\theta_{1}\right)} \frac{\sum_{t=1}^{T} x_{t}}{\sum_{t=1}^{T} x_{t}^{2}} \cdot \prod_{t=1}^{T}\left(p_{t} \cdot f_{t}\left(x_{t}\right)\right) d x_{1} \cdots d x_{T} \\
& =\prod_{t=1}^{T} p_{t} \cdot E\left(\frac{\sum_{t=1}^{T} x_{t}}{\sum_{t=1}^{T} x_{t}^{2}} \cdot \prod_{t=1}^{T} I\left(x_{t}>0\right)\right) \geq \prod_{t=1}^{T} p_{t} \cdot E\left(\frac{1}{\sum_{t=1}^{T} x_{t}} \cdot \prod_{t=1}^{T} I\left(x_{t}>0\right)\right)=\infty .
\end{aligned}
$$

The last inequality follows because $\left(\sum_{t=1}^{T} x_{t}\right)^{2} \cdot \prod_{t=1}^{T} I\left(x_{t}>0\right) \geq\left(\sum_{t=1}^{T} x_{t}^{2}\right)$. $\prod_{t=1}^{T} I\left(x_{t}>0\right)$. The second to last equality follows from the definition of joint distribution of $\left(x_{t}, \eta_{t}\right)$ and the last equality follows from Lemma 1.

For the negative part,

$$
\begin{aligned}
& E\left(\hat{\beta}_{T}^{-}\right)=E\left(\frac{\sum_{t=1}^{T} \eta_{t} x_{t}}{\sum_{t=1}^{T} x_{t}^{2}}\right)^{-}=E\left(\frac{\left(\sum_{t=1}^{T} \eta_{t} x_{t}\right)^{-}}{\sum_{t=1}^{T} x_{t}^{2}}\right) \leq E\left(\frac{\sum_{t=1}^{T}\left(\eta_{t} x_{t}\right)^{-}}{\sum_{t=1}^{T} x_{t}^{2}}\right) \\
& =\sum_{t=1}^{T} E\left(\frac{\left(\eta_{t} x_{t}\right)^{-}}{\sum_{t=1}^{T} x_{t}^{2}}\right) \leq \sum_{t=1}^{T} E\left(\frac{\left(\eta_{t} x_{t}\right)^{-}}{x_{t}^{2}}\right)=\sum_{t=1}^{T} E\left(\frac{\eta_{t}}{x_{t}}\right)^{-}<\infty .
\end{aligned}
$$

The last equality follows by explicitly writing the expectation $E(\cdot)$ out.

\section{Further discussion}

Some comments: 
1. In our example, since $x_{t}$ and $\eta_{t}$ each has compact support, each has moments of all order.

2. The assumption that the median of $x_{t}$ is zero is to simplify the algebra. We could instead choose a value $c\left(\theta_{t}\right)$ such that a given quantile of $x_{t}$ is zero.

3. Similarly, it was for simplicity that we used discreteness in part of the conditional distribution of $\eta_{t}$ given $x_{t}$. Our result still holds if $\eta_{t}$ is continuous around each of the discrete values used above.

4. Much of the complication of the example is because we set ourselves the goals of $E\left(\hat{\beta}_{T}-\beta\right)=\infty$, which requires $E\left(\hat{\beta}_{T}-\beta\right)^{+}=\infty$ and $E\left(\hat{\beta}_{T}-\beta\right)^{-}<\infty$. Had we aimed solely for $E\left|\hat{\beta}_{T}-\beta\right|=\infty$, we could have accomplished this with the same process for $x_{t}$ but with $\eta_{t}$ simply an i.i.d. Bernoulli process.

5. In our example, as $x \rightarrow 0, P\left(x_{t}^{2} \leq x\right)=P\left(\left|x_{t}\right| \leq \sqrt{x}\right)=\frac{1}{1-\theta_{t}} x^{\left(1-\theta_{t}\right) / 2}=$ $O\left(x^{-\delta_{t} / 2}\right)$. By construction, we have $\sum_{t=1}^{T} \delta_{t} \leq 1$, so $\sum_{t=1}^{T} \delta_{t} / 2 \leq \frac{1}{2}$. It obviously violates the sufficient condition in Lemma 2 , which requires $\sum_{t=1}^{T} \delta_{t} / 2>1$.

6. In the notation of Lemma 1, Sriram and Bose (1988) considered $E\left(\sum_{t=1}^{T} z_{t}\right)^{-1}$ with constant $\delta$ (i.e., $\delta_{t}=\delta<1$ for all $t$ ). They showed $E\left(\sum_{t=1}^{T} z_{t}\right)^{-1}=\infty$ for $T$ sufficiently small, $E\left(\sum_{t=1}^{T} z_{t}\right)^{-1}<\infty$ for $T$ sufficiently large. As in Stuttgen (2014), our Lemma 1 allows the density of $z_{t}$ to shift with $t$, with $\delta_{t}$ becoming smaller and the density becoming steeper as $t$ increases. The first part of the proof of Lemma 1 is completely different from the proof in Sriram and Bose (1988).

7. As noted below Lemma 1, we only require a symmetric Lipschitz condition of $P\left(x_{t}^{2} \leq x\right)$ at origin. This contrasts with the stronger assumption in Findley and Wei (2002), which, when specialized to the univariate case, imposes a uniform Lipschitz condition on $\mathbb{R}$. 


\section{Appendix}

Details on proof of Lemma 1:

Here is the proof that $\sum_{t=1}^{T} \delta_{t}>s \Rightarrow E\left(\sum_{t=1}^{T} z_{t}\right)^{-s}<\infty$.

Assume $\sum_{t=1}^{T} \delta_{t}>s$. We mimic the argument made in Sriram and Bose (1988) for constant $\delta$.

Let $S_{T}=\sum_{t=1}^{T} z_{t}$. Then

$$
\begin{aligned}
E\left(1 / S_{T}^{s}\right) & =\int_{0}^{\infty} P\left[1 / S_{T}^{s} \geq x\right] d x=\int_{0}^{\infty} P\left[e^{-x S_{T}^{s}} \geq e^{-1}\right] d x \leq e \int_{0}^{\infty} E\left[e^{-x^{1 / s} S_{T}}\right] d x \\
& =e\left(\int_{0}^{1}+\int_{1}^{\infty}\right) \prod_{t=1}^{T} E\left[e^{-x^{1 / s} z_{t}}\right] d x
\end{aligned}
$$

where the inequality follows from Markov inequality and the last equality follows from the independence of $z_{t}$ 's. Clearly $\int_{0}^{1} \prod_{t=1}^{T} E\left[e^{-x^{1 / s} z_{t}}\right] d x<\infty$. For $1<$ $x<\infty$, we have,

$$
\begin{aligned}
E e^{-x^{1 / s} z_{t}} & =\int_{0}^{1} P\left[e^{-x^{1 / s} z_{t}} \geq y\right] d y=\int_{0}^{1} P\left[z_{t} \leq-(\log y) / x^{1 / s}\right] d y \\
& =x^{1 / s} \int_{0}^{\infty} P\left[z_{t} \leq z\right] e^{-z x^{1 / s}} d z \leq x^{1 / s} \int_{0}^{\infty} z^{\delta_{t}} e^{-z x^{1 / s}} d z=C \cdot x^{-\delta_{t} / s}
\end{aligned}
$$

where the third and last equality follow for change of variable and the inequality follows from the fact $P\left(z_{t} \leq z\right)=z^{\delta_{t}}<1$. Here $C$ is a generic constant.

All together, we have

$E\left(\sum_{t=1}^{T} z_{t}\right)^{-s} \leq e\left(\int_{0}^{1}+\int_{1}^{\infty}\right) \prod_{t=1}^{T} E\left[e^{-x^{1 / s} z_{t}}\right] d x \leq C_{1}+C \int_{1}^{\infty} x^{-\sum_{t=1}^{T} \delta_{t} / s} d x<\infty$,

since $\sum_{t=1}^{T} \delta_{t}>s$. 


\section{References}

Bao, Yong and Aman Ullah, 2010, "Expectation of quadratic forms in normal and nonnormal variables with applications," Journal of Statistical Planning and Inference $\underline{140}, 1193-1205$.

Findley, David F. and Ching-Zong Wei, 2002, "AIC, Overfitting Principles, and the Boundedness of Moments of Inverse Matrices for Vector Autoregressions and Related Models," Journal of Multivariate Analysis 3, 415-450.

Khuri, André and George Casella, 2002, "The Existence of the First Negative Moment Revisited," The American Statistician ㅁ6, 44-47.

Shaman, Paul, 2010, "Generalized Levinson-Durbin sequences, binomial coefficients and autoregressive estimation," Journal of Multivariate Analysis 101, 1263-1273.

Sriram, T. N. and Arup Bose, 1988, "Sequential shrinkage estimation in the general linear model," Sequential Analysis: Design Methods and Applications 7, 149-163.

Stuttgen, Luke, 2014, "Considerations of Unbounded Bias in Least Squares Estimators," unpublished senior honors thesis, University of Wisconsin. 